Service social

\title{
La recherche évaluative dans le réseau de la santé et des services sociaux en région éloignée. L'expérience de définition d'une évaluation du travail de rue en Abitibi-Témiscamingue
}

\section{Paule Simard et Nicole Berthiaume}

Volume 47, numéro 1-2, 1998

Évaluation - Colloque 1999

URI : https://id.erudit.org/iderudit/706788ar

DOI : https://doi.org/10.7202/706788ar

Aller au sommaire du numéro

Éditeur(s)

École de service social de l'Université Laval

ISSN

1708-1734 (numérique)

Découvrir la revue

Citer cet article

Simard, P. \& Berthiaume, N. (1998). La recherche évaluative dans le réseau de la santé et des services sociaux en région éloignée. L'expérience de définition d'une évaluation du travail de rue en Abitibi-Témiscamingue. Service social, 47(1-2), 191-210. https://doi.org/10.7202/706788ar
Résumé de l'article

Il existe actuellement un courant propice à l'évaluation dans le réseau de la santé et des services sociaux québécois. Toutefois, en raison du contexte structurel et légal dont doivent tenir compte les régies régionales, l'évaluation tend à répondre beaucoup plus à une logique de planification et de gestion qu'à une logique d'action préoccupée par les besoins des intervenants et des populations visées. Cet article vise à démontrer que, malgré les nombreux obstacles rencontrés, il est pertinent de concevoir des formes d'évaluation qui tiennent compte des intérêts des différents acteurs concernés. Il est ainsi possible de réaliser des évaluations lorsque certaines conditions sont réunies. Le projet d'évaluation du travail de rue à Rouyn-Noranda en est un exemple. 


\title{
La recherche évaluative dans le réseau de la santé et des services sociaux en région éloignée L'expérience de définition d'une évaluation du travail de rue en Abitibi-Témiscamingue
}

\author{
Paule SIMARD \\ Nicole BERTHIAUME
}

II existe actuellement un courant propice à l'évaluation dans le réseau de la santé et des services sociaux québécois. Toutefois, en raison du contexte structurel et légal dont doivent tenir compte les régies régionales, l'évaluation tend à répondre beaucoup plus à une logique de planification et de gestion qu'à une logique d'action préoccupée par les besoins des intervenants et des populations visées. Cet article vise à démontrer que, malgré les nombreux obstacles rencontrés, il est pertinent de concevoir des formes d'évaluation qui tiennent compte des intérêts des différents acteurs concernés. II est ainsi possible de réaliser des évaluations lorsque certaines conditions sont réunies. Le projet d'évaluation du travail de rue à Rouyn-Noranda en est un exemple.

Currently, there is a favorable trend towards evaluation in the health and social services network in Québec. However, due to the structural and legal context which must be taken into account by the regional boards, evaluation tends to respond to a logic of planning and administration gestion rather than to an action logic that would meet the workers and the populations needs. This paper wishes to illustrate the importance of developing types of evaluation that would take into account the interests of the diverse actors involved. It is possible to do such evaluation when some basic conditions are respected. This is illustrated through the example of the evaluation project of outreachwork in Rouyn-Noranda. 
L'évaluation est un sujet d'actualité dans le réseau de la santé et des services sociaux, notamment dans les régies régionales. En effet, depuis plusieurs années, et surtout depuis la réforme du système de santé, l'évaluation est devenue une dimension incontournable du mandat des régies régionales. En dépit, ou en raison, de cet engouement, le champ de l'évaluation est parfois peu connu et souvent mal cerné. II reste donc beaucoup à faire pour passer du discours à une pratique articulée et cohérente.

Notre propos concernant l'évaluation en région éloignée vise à montrer que, dans la gamme des diverses formes d'évaluation, celles qui permettent de répondre aux questions des intervenants et des populations visées sont souvent négligées. II faut dire que les contraintes pour réaliser des évaluations sont importantes en région éloignée. Néanmoins, il se fait des évaluations intéressantes. Nous en témoignerons en rapportant un projet de recherche évaluative que nous avons réussi à mettre en place en Abitibi-Témiscamingue, celui de l'évaluation du travail de rue à Rouyn-Noranda.

\section{L'ÉVALUATION DANS LE RÉSEAU DE LA SANTÉ ET DES SERVICES SOCIAUX}

Dans la Loi sur les services de santé et les services sociaux, il est stipulé que « la Régie régionale a principalement pour objet de planifier, d'organiser, de mettre en œuvre et d'évaluer, dans la région, les programmes de santé et de services sociaux élaborés par le ministre " (art. 340). Il est également dit que la Régie régionale doit évaluer "l'efficacité des services de santé et des services sociaux, le degré d'atteinte des objectifs poursuivis et le degré de satisfaction des usagers à l'égard des services " et qu'elle doit " élaborer et mettre en œuvre, conformément aux directives du ministre, des évaluations de programmes et de services auxquels participent les établissements " (art. 346).

Outre les aspects légaux de la question, d'autres facteurs viennent rendre plus complexe le portrait de l'évaluation. C'est le cas notamment de toutes les demandes concernant l'évaluation, généralement sous forme d'indicateurs de suivi, qui accompagnent les orientations nationales en matière de santé et de services sociaux. 
La Politique de la santé et du bien-être précise l'importance de « monitorer les activités et évaluer les interventions " par un suivi des objectifs (développement d'indicateurs et de systèmes d'information « clientèle " et "population ", évaluation systématique et continue de la qualité et de l'efficacité des services) et le développement des connaissances (recherche sur les facteurs de protection et de promotion, développement de la recherche évaluative et de la recherche-action) (MSSS, 1997, p. 180).

Par ailleurs, les Priorités nationales de santé publique : 1997-2002 prévoient « un processus continu de suivi » dont l'« objectif est de transmettre régulièrement de l'information aux acteurs concernés, de susciter une mobilisation et de permettre d'effectuer les ajustements nécessaires " (MSSS, 1997, p. 86). II s'agit principalement d'une évaluation des résultats qui permettra de « répondre à deux grands ensembles de questions, l'un portant sur les principes directeurs qui doivent orienter de façon majeure les interventions, et l'autre, sur l'atteinte des résultats escomptés au regard des priorités 》 (idem).

S'ajoutent à ces politiques nationales d'autres commandes qui posent des balises supplémentaires au mandat d'évaluation des régies régionales, notamment : l'allocation des ressources, les compressions budgétaires, la reddition de compte à la commission parlementaire des affaires sociales et les ententes de gestion que vient d'instituer la nouvelle ministre.

L'ensemble de ces consignes (ou contraintes) légales et contextuelles crée un environnement propice à l'évaluation, mais lui fournit des balises spécifiques qui l'orientent principalement vers une logique gestionnaire ou de planification, c'est-à-dire qui sert avant tout les intérêts des décideurs et des planificateurs (Mercier, 1996, p. 89) en cherchant à définir la pertinence des services offerts et à mesurer les résultats et les impacts, de même que l'efficacité et l'efficience des interventions. Dans cette perspective, la finalité de l'évaluation est de savoir si, avec l'argent investi, on obtient les résultats escomptés.

Pourtant, la complexité des interventions sociales mises en place par le réseau de la santé et des services sociaux fait en sorte que les évaluations n'arrivent pas à démontrer la chaîne causale reliant les objectifs définis et les interventions à l'état de santé ou de bien-être de la population (Brunelle et Saucier, 1999). En effet, dans les domaines 
de la santé et du social, il apparaît particulièrement difficile de prouver, même à partir d'une approche expérimentale, que tel résultat atteint est dû à telle intervention ou à tel ensemble d'interventions en particulier.

La logique gestionnaire cherche à savoir si l'intervention planifiée a été mise en œuvre comme il avait été prévu. II s'agit alors de savoir si les objectifs de départ ont été atteints. Bien que cette approche ait le mérite de démontrer si l'on s'est bien rendu où l'on voulait aller, elle ne dit cependant rien sur le cheminement qui permet (ou parfois ne permet pas) d'aller du point A (les objectifs de départ) au point B (l'atteinte des objectifs). II peut arriver que des objectifs ne soient pas atteints, et il est alors important de le savoir. Toutefois, il apparaît beaucoup plus constructif de savoir pourquoi ils n'ont pas été atteints que de savoir simplement qu'ils ne l'ont pas été. D'où la pertinence des évaluations de processus qui visent à montrer les étapes du cheminement poursuivi et les mécanismes qui sous-tendent ce cheminement.

Cela nous amène, comme le propose le courant d'évaluation instauré par Patton (1986) et Guba et Lincoln (1989), à une évaluation centrée sur l'action, au service de l'action. Ce type d'évaluation vise en fait à traduire en questions d'évaluation les préoccupations des principales personnes concernées par le programme ou l'intervention que l'on veut étudier, surtout les intervenants et les clientèles visées. Cela nous amène également à nous préoccuper de la satisfaction des clients dans une évaluation. Car, comme le souligne Joubert, " peu importe le type ou les questions d'évaluation, dans la mesure où l'on vise à impliquer les clients dans le processus même de l'intervention, c'est finalement le degré de satisfaction des clientèles à l'endroit du programme que l'évaluation vise à mesurer " $(1990$, p. 4).

Mais qu'en est-il, au-delà des intentions, des activités d'évaluation dans les régies régionales, spécifiquement dans celles des régions éloignées? Quelles sont les contraintes et les opportunités qu'entraîne un tel contexte? Quelles sont les avenues à explorer? C'est à ces questions que nous tenterons de répondre à partir d'un exemple concret, celui de l'Abitibi-Témiscaminque. 


\section{L'ÉVALUATION DANS UNE RÉGION ÉLOIGNÉE LE CAS DE L'ABITIBI-TÉMISCAMINGUE}

Le contexte actuel, pour les régies régionales des régions éloignées, n'est pas très propice à la réalisation d'évaluations, malgré un climat général qui, comme on l'a souligné précédemment, est plutôt favorable à l'évaluation. De nombreuses contraintes viennent limiter la réalisation d'évaluations et orienter les quelques-unes qui peuvent être produites. Malgré tout, il existe aussi quelques avantages qu'il faudrait savoir exploiter, de même que plusieurs pistes de solution.

\section{Un manque de ressources humaines}

Un des plus grands défis à surmonter en Abitibi-Témiscamingue est le manque de ressources humaines qualifiées pour faire les évaluations. Cette contrainte est triple.

Premièrement, les équipes de recherche et d'évaluation sont restreintes. Dans la région, il existe deux équipes de recherche et d'évaluation rattachées respectivement à la Direction des programmes et des services (deux agents de recherche) et à la Direction de la santé publique (trois agents de recherche).

Deuxièmement, ces professionnels sont déjà surchargés par les mandats légaux de la connaissance et de la surveillance ou du suivi de gestion. Aux programmes et services, les activités d'évaluation portent principalement sur l'implantation des services de santé et des services sociaux. En santé publique, ce sont surtout les études de clientèles (ligne info-suicide, couverture vaccinale, etc.) et les études épidémiologiques en santé physique (chlamydiose, par exemple) qui ont été développées. II s'y fait peu d'évaluations en dehors de activités de monitorage. En fait, les évaluations se limitent habituellement aux évaluations d'implantation prévues dans la planification des interventions. La recherche évaluative n'y est pas monnaie courante.

Troisièmement, l'expertise en évaluation n'est pas très poussée. Les professionnels démontrent cependant un intérêt manifeste pour se former et développer ce champ d'expertise qui, pour beaucoup, est nouveau. Toutefois, dans les faits, il s'avère difficile pour eux de bénéficier de formations et de participer à des forums ou à des comités provinciaux pour parfaire leur expertise en évaluation. En effet, les 
déplacements à partir de l'Abitibi-Témiscamingue sont très coûteux, ce qui restreint la mobilité du personnel. De plus, les professionnels en évaluation des régions éloignées ne disposent que de peu de temps pour se former ou assister à des événements provinciaux (comités, colloques, etc.).

En théorie, le mandat d'évaluation n'est pas réservé aux seuls agents de recherche. Dans la pratique, cependant, les professionnels en planification et en programmation, même s'ils ont aussi un mandat de recherche et d'évaluation, sont débordés par la mise en place d'interventions. Ils se limitent donc au suivi de gestion des principaux éléments de leurs programmes respectifs.

Par ailleurs, il y a un manque de ressources humaines qualifiées en recherche et en évaluation dans la région. C'est pourquoi les procédures de gestion du Programme de subvention en santé publique de l'Abitibi-Témiscamingue ont été modifiées en s'inspirant du modèle de la Gaspésie-Îles-de-la-Madeleine. Dorénavant, les organismes ou les établissements du réseau doivent présenter des idées de recherche qui seront réalisées par des professionnels de la Direction de la santé publique en collaboration avec les promoteurs plutôt que par des chercheurs indépendants. Cette décision a été prise après qu'on eut constaté les lacunes méthodologiques de plusieurs projets réalisés dans les établissements ou les organismes communautaires, déficiences surtout attribuées aux difficultés de recrutement de chercheurs qualifiés.

\section{Le financement}

Une autre difficulté à laquelle doit faire face l'Abitibi-Témiscamingue est le financement des évaluations. Si plusieurs programmes provinciaux prévoient des sommes pour l'évaluation, il n'en demeure pas moins que les interventions réalisées à l'échelle régionale ou locale ne disposent pas toujours de budgets pour l'évaluation.

En ce qui a trait plus spécifiquement à la recherche évaluative, cette forme d'évaluation commande des moyens financiers considérables et les exigences des bailleurs de fonds sont plus restrictives. Par exemple, les subventions sont attribuées à des chercheurs qui possèdent un doctorat; or, ceux-ci sont plutôt rares dans le réseau de la santé et des services sociaux d'Abitibi-Témiscamingue. 
De plus, les bailleurs de fonds exigent, pour financer des projets de recherche, la constitution de partenariats et d'équipes de recherche regroupant des chercheurs de diverses provenances. En AbitibiTémiscamingue, les chercheurs ne sont pas nécessairement nombreux, bien qu'il y ait un certain bassin de chercheurs grâce à la présence d'une composante de l'Université du Québec. Ce type de partenariat offre néanmoins de bonnes opportunités de part et d'autre de réaliser des évaluations. Le défi est alors de trouver un point de rencontre entre les intérêts des chercheurs universitaires et ceux des différents partenaires du réseau de la santé et des services sociaux.

D'autres sources de financement à des fins d'évaluation sont parfois disponibles à partir de fonds spécifiques (par exemple, le Fonds pour l'adaptation des services de santé) et les exigences sont alors moins sévères que dans le cas de la recherche subventionnée. Pourtant, même là, il arrive que des professionnels doivent laisser passer des opportunités parce qu'ils n'ont pas le temps d'élaborer un projet.

\section{Les relations entre le Ministère et les régions}

À ces contraintes liées aux ressources de la régie régionale s'ajoutent des enjeux d'ordre politique qui touchent aux relations entre les niveaux provincial et régional. Avec la transformation du réseau, et surtout la mise en place d'ententes de gestion entre les régies et le ministère de la Santé et des Services sociaux (MSSS), les attentes du niveau provincial en matière d'évaluation s'orientent surtout vers la reddition de compte au moyen d'indicateurs de gestion.

En outre, plusieurs évaluations sont prévues à même des programmes provinciaux et les procédures sont décidées au MSSS. Les régions ne font alors que les appliquer, avec ou sans l'aide de ressources provinciales. Dans ce contexte, les régions ne sentent pas toujours que leurs besoins sont pris en considération. II en est de même pour les grandes orientations du MSSS, notamment les Priorités nationales de santé publique, pour lesquelles le Ministère a prévu une procédure d'évaluation. Lors d'un séminaire tenu durant les Journées annuelles de santé publique de 1998, plusieurs régions ont exprimé leur malaise quant à une évaluation quantitative des résultats obtenus qui ne dise rien des contextes régionaux spécifiques pouvant expliquer leur «performance » respective. Ces commentaires n'ont toutefois pas 
amené de modifications notables, du moins à court terme, dans la procédure provinciale d'évaluation.

\section{Au service des besoins du milieu}

Un autre défi important de l'évaluation dans les régies régionales consiste à réaliser des évaluations qui répondent adéquatement aux besoins du milieu. Des efforts sont faits en Abitibi-Témiscamingue pour trouver des moyens de prendre en compte les intérêts des différents partenaires, établissements et organismes communautaires, dans la réalisation des évaluations. Toutefois, dans l'ensemble, les évaluations portent tout de même sur les préoccupations des gestionnaires et des planificateurs de la Régie régionale, à savoir si le service ou le programme a été implanté comme prévu ou si les modalités d'implantation de la mesure sont conformes aux objectifs visés (Équipe La Petite Rencontre de Val-d’Or et collab., 1998; RSSSAT, 1999, 1996 et 1995).

II faut aussi se rappeler que l'évaluation dans le domaine de la santé et des services sociaux n'est pas une chasse gardée des régies régionales. Les établissements et les organismes communautaires doivent aussi évaluer leurs interventions. Or, pour les intervenants des établissements du réseau, la question de l'évaluation suscite des réactions très diverses et parfois divergentes. D'une part, ils considèrent l'évaluation comme utile à leur travail, sans toutefois avoir le temps, l'argent ni toujours le savoir-faire pour la réaliser. D'autre part, et c'est là le cœur de la question, les intervenants ne connaissent souvent pas très bien les différentes formes d'évaluation. Aussi, de manière générale, perçoivent-ils l'évaluation comme un outil de contrôle de leur travail.

Les enjeux sont les mêmes pour les organismes communautaires du réseau, mais ils se manifestent de manière plus pointue. Les organismes communautaires ne sont généralement pas opposés à l'évaluation, mais ils voudraient la faire eux-mêmes de façon qu'elle réponde plus adéquatement à leurs besoins (Midy et collab., 1998) et, surtout, qu'elle soit respectueuse de leur spécificité (Comité ministériel sur l'évaluation, 1997). Lorsqu'elle est prévue ou imposée par une régie régionale, l'évaluation est considérée comme un moyen de contrôler leur travail (Parazelli, 1990). 
Malgré les embûches qu'elles rencontrent dans la réalisation d'évaluations, les régions éloignées possèdent tout de même certains avantages sur celles des grands centres, avantages qu'elles doivent mettre à profit. En Abitibi-Témiscamingue, par exemple, les professionnels en planification et en programmation, de même que les agents de recherche, peuvent être plus directement en contact avec les établissements et les groupes communautaires du réseau de la santé et des services sociaux. En effet, il n'y a souvent qu'un porteur de dossier à la Régie régionale pour toute la région et celui-ci entretient généralement des liens avec plusieurs établissements et organismes communautaires. II peut ainsi s'établir des arrimages intéressants dans une perspective d'évaluation ou de recherche évaluative. Ce rapprochement peut également permettre de développer des formes d'évaluation adaptées et utiles aux intervenants du terrain tout en servant les fins de mesure des effets à court terme qui préoccupent davantage la Régie régionale.

\section{La nécessité de développer des outils d'évaluation}

À 'occasion d'une tournée réalisée auprès des établissements de type CLSC et certains organismes communautaires dans le cadre de la définition du Plan de mise en œuvre des Priorités nationales de santé publique en Abitibi-Témiscamingue, il est ressorti qu'un des obstacles à la réalisation d'évaluations est le manque d'outils d'évaluation. II y aurait donc un effort à faire pour instrumenter les établissements afin qu'ils réalisent eux-mêmes des évaluations. En Abitibi-Témiscamingue, l'appui fourni par la Régie régionale aux établissements et aux organismes communautaires s'est traduit jusqu'à maintenant surtout en termes de données statistiques et, à l'occasion, par un support méthodologique pour l'élaboration de leurs projets d'évaluation.

Enfin, la Régie régionale de l'Abitibi-Témiscamingue entend, à l'instar d'autres régies régionales telles Lanaudière et Québec, élaborer au cours des prochains mois un cadre de référence en évaluation. Un tel outil devrait définir les différentes formes d'évaluation et proposer des orientations pour les réaliser. II pourrait également spécifier les différentes responsabilités quant à l'évaluation, ce qui permettrait d'établir les besoins en matière d'outils ou de support méthodologique.

Pour illustrer de manière plus concrète les enjeux de l'évaluation en région éloignée, nous ferons appel à une expérience de recherche 
actuellement en cours en Abitibi-Témiscamingue, celle de l'évaluation du programme “ Travail de rue " à Rouyn-Noranda ${ }^{1}$. Nous analyserons le processus qui a permis à un organisme communautaire et à la Direction de la santé publique de l'Abitibi-Témiscamingue de définir conjointement l'objet d'évaluation et la méthodologie en vue d'obtenir un financement.

\section{L'ÉVALUATION DU PROGRAMME «TRAVAIL DE RUE » EN ABITIBI-TÉMISCAMINGUE}

À Rouyn-Noranda, le programme «Travail de rue » existe de façon continue depuis novembre 1995, moment où un organisme communautaire, Arrimage Jeunesse, a embauché un travailleur de rue. Depuis avril 1997, le financement du programme est assuré par la Régie régionale de la santé et des services sociaux de l'AbitibiTémiscamingue, permettant ainsi le maintien de deux travailleurs de rue sur le terrain. La Régie régionale, toujours en collaboration avec Arrimage Jeunesse, soutient également l'implantation du programme dans les autres municipalités régionales de comté (MRC) de la région (Abitibi-Ouest, Abitibi, Vallée-de-l'Or et Témiscamingue).

Arrimage Jeunesse est un organisme sans but lucratif, dont les deux seuls salariés sont les travailleurs de rue. L'équipe de supervision est bénévole. Après quatre années de fonctionnement, l'équipe d'Arrimage Jeunesse, travailleurs de rue et membres du conseil d'administration et du comité de supervision, a manifesté de l'intérêt pour évaluer le programme de travail de rue. Toutefois, comme les ressources au sein de l'organisme ne permettaient pas de réaliser une telle évaluation, ils ont demandé l'appui de la Direction régionale de la santé publique.

Au printemps 1998, une première occasion consistant à déposer une demande au Fonds pour l'adaptation des services de santé (fédéral) est écartée. Le temps disponible pour rédiger la demande est très court et il n'y a pas, à la Direction de la santé publique, de professionnels disponibles et qualifiés pour le faire.

1. Recherche évaluative financée par le Conseil québécois de la recherche sociale (projet RS-3301). 
À l'été 1998, la Direction de la santé publique embauche un professionnel pour développer la recherche sociale et évaluative. Cette personne détenant un doctorat, elle se qualifie donc pour faire une demande de subvention auprès d'organismes qui financent la recherche. II est alors convenu qu'une demande serait soumise au Conseil québécois de la recherche sociale (CQRS) pour réaliser l'évaluation du programme de travail de rue à Rouyn-Noranda.

Pour ce faire, une équipe de recherche est constituée. Au début, celleci est composée d'un chercheur de la Direction de la santé publique, du responsable régional de l'implantation du programme « Travail de rue " en Abitibi-Témiscamingue, de deux membres du conseil d'administration d'Arrimage Jeunesse (également superviseurs cliniques des travailleurs de rue) et des deux travailleurs de rue. En cours de route, s'ajoutent également deux chercheurs, un de l'Université du Québec en Abitibi-Témiscamingue et un autre du Centre de santé publique de Québec.

Dès le départ, il va de soi qu'il s'agit d'une recherche en partenariat, dans laquelle tous les membres de l'équipe collaborent à la définition et à la réalisation de l'ensemble des étapes de la recherche. Toutefois, étant donné le peu de temps dont dispose l'équipe pour rédiger la demande (environ deux mois), la période d'apprivoisement entre le chercheur principal et les gens de terrain, qui eux se connaissent, se révèle une peu courte pour comprendre et débattre à fond des besoins et des intérêts de chacun. Malgré cela, il est possible de préciser des objectifs et des orientations qui rallient toute l'équipe.

La méthodologie de la recherche est également construite dans un va-et-vient entre les chercheurs et les autres membres de l'équipe de manière à trouver la façon la plus harmonieuse et efficace d'assurer l'arrimage entre les exigences scientifiques d'une recherche et les besoins des intervenants sur le terrain. 


\section{Figure 1}

\section{Les objectifs du projet d'évaluation du travail de rue à Rouyn-Noranda}

\section{But}

Améliorer l'arrimage entre le programme " Travail de rue » et les différents niveaux d'intervenants et d'intervenantes des réseaux institutionnels et communautaires de manière à apporter un soutien plus complet aux jeunes de la rue.

\section{Objectif général 1}

Comprendre la structure de fonctionnement du programme « Travail de rue » en ce qui a trait aux relations entre les travailleurs de rue et les ressources du milieu.

\section{Objectifs spécifiques}

- Montrer comment le programme « Travail de rue " s'insère dans la géographie locale des différents milieux d'intervention (réseau de la santé et des services sociaux, éducation, emploi, justice) travaillant sur des problématiques touchant les jeunes de la rue (toxicomanie, violence, prostitution, maladies transmissibles sexuellement, criminalité).

- Cerner les mécanismes qui sous-tendent les liens qui se créent (ou ne se créent pas) et les dynamiques de collaboration entre les travailleurs de rue et les différents niveaux d'intervenants et d'intervenantes institutionnels et communautaires.

\section{Objectif général 2}

Évaluer les effets immédiats du programme « Travail de rue ».

\section{Objectif spécifique}

- Cerner le parcours des jeunes dans la chaîne des services offerts aussi bien par les travailleurs de rue que par les intervenants auxquels ils sont envoyés.

\section{Objectif général 3}

À partir des conclusions de l'évaluation du programme, développer des outils pour appuyer la mise en place et le suivi de programmes « Travail de rue » dans la région.

\section{Objectifs spécifiques}

- Identifier, avec les différents intervenants et intervenantes, des indicateurs de suivi du programme qui permettront de fournir, de manière continue, un portrait qualitatif de la situation.

- Développer des outils de suivi et d'évaluation.

- Réaliser un outil de formation et d'implantation s'adressant aux travailleurs de rue et aux comités d'implantation de nouveaux programmes. 
Une réponse positive pour le financement est reçue en avril 1999 et le projet démarre en juin de la même année. L'agent de recherche prévu dans le protocole est sélectionné par l'ensemble de l'équipe et les travailleurs de rue jouent un rôle important dans la définition des critères de sélection; la personne doit être bien reçue par les jeunes qui fréquentent les travailleurs de rue.

À cette étape de démarrage, les discussions de l'équipe portent beaucoup sur les aspects méthodologiques de la recherche, surtout en ce qui a trait aux stratégies de recrutement des informateurs, particulièrement des jeunes. Après avoir constaté que le recrutement des jeunes est un peu plus difficile que ce qui avait été envisagé au départ, il est convenu que l'agent de recherche suive, quelques après-midi par semaine, les travailleurs de rue dans leur visite des lieux de rencontre des jeunes. Les jeunes disposés à participer sont alors interviewés sur place de manière à profiter de l'occasion car, dès le début, il apparaît que plusieurs rendez-vous ne sont pas respectés.

Il est également convenu que l'on retiendra les méthodes de collecte qui ont le moins d'impact sur la pratique des travailleurs de rue et, surtout, sur les relations établies entre les jeunes et les travailleurs de rue.

\section{Les enjeux de cette évaluation}

Un des principaux défis auxquels la recherche a dû faire face, et c'est là un des enjeux majeurs de la recherche évaluative sur les interventions sociales dans le réseau de la santé et des services sociaux, est la définition des objectifs et de l'objet d'évaluation. Pour illustrer ces enjeux, nous tenterons de mettre en lumière les intérêts de chaque groupe d'acteurs.

Prenons d'abord les intérêts de l'organisme responsable du projet. II faut se rappeler que c'est l'organisme responsable du travail de rue, Arrimage Jeunesse, qui manifeste en premier le désir d'évaluer son programme. Pour l'organisme, l'intérêt est triple. Premièrement, il s'agit de voir si ses modes d'intervention peuvent être améliorés (évaluation de processus). L'évaluation doit donc porter sur la pratique mise en œuvre par les travailleurs de rue et par l'équipe de supervision pour savoir si c'est la meilleure façon de rejoindre la clientèle visée.

Deuxièmement, il y a également un intérêt à démontrer les effets du travail de rue (évaluation d'impact). Arrimage Jeunesse veut savoir si 
le travail de rue a vraiment des effets bénéfiques sur les personnes qu'il rejoint, notamment sur leur capacité à prendre en main leur santé et leur bien-être. L'évaluation d'impact revêt également un caractère stratégique, puisque l'organisme vise un financement à long terme de son programme et les bailleurs de fonds cherchent généralement à savoir si les programmes financés ont réellement un effet sur la population.

Troisièmement, Arrimage Jeunesse pense que l'évaluation du programme par l'intermédiaire d'un projet spécifique lui permettra d'élaborer des outils de suivi continu.

En ce qui a trait plus spécifiquement à l'intérêt des travailleurs de rue dans la réalisation de l'évaluation, ceux-ci souhaitent que la recherche permette de saisir le degré de satisfaction des jeunes à l'égard de leur intervention.

Pour la Direction de la santé publique, l'intérêt de ce projet est double. D'une part, il doit permettre de fournir des éléments propres à soutenir l'implantation du programme "Travail de rue " dans les autres MRC de la région. D'autre part, le projet vise, et c'est là un objectif commun avec Arrimage Jeunesse, à montrer la pertinence du programme pour confirmer un financement plus substantiel2.

Pour le chercheur de la Direction de la santé publique, l'intérêt premier de cette évaluation est qu'elle soit directement au service de l'action (Patton, 1986). Un autre intérêt qu'il y voit est qu'elle tente de répondre aux exigences scientifiques et logistiques du bailleur de fonds ciblé (ici le CQRS). II s'agit donc, entre autres, de s'assurer que l'objet de recherche soit novateur par rapport aux autres recherches portant sur le travail de rue, ce qui n'était, en somme, pas très difficile puisque peu de projets ont été réalisés sur cette pratique.

De manière générale, les évaluations du travail de rue qui ont été produites visent principalement à dresser un portrait des utilisateurs (Beauchemin et collab., 1994; General Accounting Office, 1993; Freudenberg, 1995; Noël et collab., 1994; School of Public Health et Institute for Health Policy Studies, 1993). Certaines évaluations ont également porté plus spécifiquement sur le processus du travail de rue, notamment son impact sur le mode de vie des travailleurs de rue

2. À l'heure actuelle, le financement est assuré pour une période de deux ans. 
ou le type d'approche utilisé pour rejoindre la clientèle (interventionniste ou non) (Moore et collab., 1998).

De plus, comme le travail de rue n'est généralement pas utilisé seul, mais qu'il s'inscrit plutôt dans un ensemble de moyens de prévention des MTS-Sida, les évaluations ont également tenté de vérifier quelles étaient les approches les plus efficaces (Janz et collab., 1996; Moore et collab., 1998; Zimmerman et collab., 1995). En ce qui concerne la pratique globale du travail de rue, telle qu'elle a été mise en œuvre par l'équipe d'Arrimage Jeunesse à Rouyn-Noranda, aucune étude ne semble avoir été réalisée sur ce type d'approche (Moore et collab., 1998; Paquet, 1998; Pharand, 1995).

En ce qui a trait aux critères organisationnels, l'équipe souhaitait associer au projet d'autres chercheurs pour assurer un soutien scientifique accru. Des contacts antérieurs de membres de la Direction de la santé publique avec une équipe du Centre de santé publique de Québec ont facilité le recrutement d'une chercheuse connaissant bien le sujet à l'étude.

Nous voulions également établir des contacts avec des chercheurs de l'UQAT. Cependant, étant donné que le chercheur principal est nouveau dans la région et que l'équipe dispose de peu de temps pour rédiger la demande, le recrutement est un peu difficile. Un professeur travaillant à des problématiques connexes a finalement accepté de se joindre à l'équipe. Son point de vue de chercheur quantitatif vient d'ailleurs ajouter une contribution tant pour la recherche elle-même que pour le suivi de l'intervention que désire faire Arrimage Jeunesse.

Un autre intérêt, dont le chercheur principal se fait le porte-parole, est la place du travail de rue comme un maillon du réseau de la santé et des services sociaux. Bien que les responsables du programme ne se considèrent pas comme faisant partie du réseau, le fait qu'ils soient financés par la Régie régionale témoigne du contraire. En fait, ce n'est pas tant l'appartenance du travail de rue au réseau de la santé qui importe, mais la complémentarité entre le travail de rue et les ressources du milieu et, plus spécifiquement, les liens de collaboration qui s'établissent ou ne s'établissent pas entre les travailleurs de rue et les ressources du milieu (à l'intérieur et à l'extérieur du réseau de la santé et des services sociaux). De plus, on voulait, au moyen de la 
recherche, comprendre le parcours des jeunes à travers les ressources du milieu à partir des informations ou des « références » faites par les travailleurs de rue.

En définitive, malgré la diversité des intérêts poursuivis par ses membres, l'équipe de recherche réussit à identifier des objectifs acceptables pour chacun d'entre eux. L'orientation générale de l'évaluation étant que le programme est là pour demeurer, il s'agit de voir comment on pourrait l'améliorer en respectant, évidemment, les besoins des groupes en présence.

Par ailleurs, il faut souligner qu'à aucun moment l'équipe ne s'est posé la question de savoir si le travail de rue constitue le meilleur moyen de rejoindre les jeunes évoluant en dehors des circuits traditionnels. Cette question, bien que pertinente, aurait donné une tout autre orientation à la recherche et ce n'était pas dans cette direction que l'équipe désirait mener sa réflexion.

\section{CONCLUSION}

En définitive, on peut dire qu'il existe un courant propice à l'évaluation dans le réseau de la santé et des services sociaux. Toutefois, compte tenu du contexte structurel et légal que connaissent les régies régionales, l'évaluation tend à répondre beaucoup plus à une logique de planification et de gestion qu'à une logique d'action préoccupée par les besoins des intervenants et des populations visées.

Pourtant, les activités du réseau de la santé et des services sociaux touchent des situations complexes qui demandent à leur tour des réponses à composantes multiples. On veut de plus en plus tenir compte des divers déterminants de la santé et agir sur ceux-ci. L'objectif est louable, mais les outils pour comprendre comment intervenir et savoir si les interventions ont porté des fruits doivent pouvoir rendre compte de cette complexité. C'est pourquoi il ne faudrait pas que l'évaluation se limite au suivi de gestion ou à la reddition de compte. II est nécessaire de développer des formes d'évaluation qui permettent aux différents groupes d'acteurs concernés de débattre, ensemble, des questions d'évaluation qui les préoccupent et non pas seulement de donner leur point de vue sur des questions formulées par les gestionnaires ou les planificateurs. 
En fait, et c'est ce que cette réflexion voulait illustrer, il y a place à l'innovation dans les façons de penser et de réaliser les évaluations. Notamment, il faut arriver à démontrer aux gestionnaires que les interventions financées ont un impact positif sur les communautés, les réseaux sociaux, l'accès aux services, etc. Or, comme les effets de ces interventions ne sont toujours pas observables à court terme, le défi est de trouver des outils pour illustrer l'évolution d'un phénomène. Évaluer l'impact d'un programme ou d'une intervention seulement à partir des résultats atteints risque de ne pas traduire toute la richesse des transformations réellement vécues par les acteurs et la communauté et qui améliorent, petit à petit, leur qualité de vie et leur santé.

L'évaluation des processus de mise en place d'interventions et de collaboration constitue donc une voie importante à laquelle des efforts devraient être consacrés aussi bien dans le réseau de la santé et des services sociaux que chez ses partenaires. Ce n'est qu'à ce prix que nous serons en mesure de comprendre les retombées réelles de nos interventions.

Paule SIMARD Agente de recherche sociosanitaire

Direction de la santé publique Régie régionale de la santé et des services sociaux de l'Abitibi-Témiscamingue

Nicole BERTHIAUME Agente de recherche et d'évaluation Direction des programmes et des services

Régie régionale de la santé et des services sociaux de l'Abitibi-Témiscamingue

\section{Références bibliographiques}

BootH, R.E. et collab. (1993). "Quantitative and Qualitative Methods to Assess Behavioral Change Among Injection Drug Users ", dans D.G. Fisher et R. Needle, AIDS and Community-Based Drug Intervention Programs : Evaluation and Outreach, The Haworth Press Inc., p. 161-183.

BRUNelLE, Y. et A. SAUCIER (1999). Les indicateurs et le système de soin, Québec, ministère de la Santé et des Services sociaux, $37 \mathrm{p}$.

CALLON, M. (1986). « Éléments pour une sociologie de la traduction ", L'année sociologique, 36, p. 169-208. 
COMITÉ MINISTÉRIEL SUR L'ÉVALUATION (1997). L'évaluation des organismes communautaires et bénévoles, Québec, ministère de la Santé et des Services sociaux, $75 \mathrm{p}$.

ÉQuipe LA PETITE Rencontre de VAL-D'Or et GRoupe SOLEIL de MALARTIC (1998). Évaluation de l'implantation du projet de soutien dans le milieu de vie pour les personnes vivant avec des troubles mentaux à Val-d'Or et Malartic, $17 \mathrm{p}$.

FREUDENBERG, N. (1995). "AIDS Prevention Strategies in the United States : A Selective Review of the Literature ", dans N. Freudenberg et M.A. Zimmerman (dir.), AIDS Prevention in the Community. Lessons from the First Decade, Washington, American Public Health Association.

General Accounting Office (1993). Needle Exchange Programs, Research Suggests Promise as an AIDS Prevention Strategy, United States General Accounting Office.

GUBA, E.G. et Y.S. LINCOLN (1989). Fourth Generation Evaluation, Newbury Park, CA, Sage Publications, $294 \mathrm{p}$.

JOUBERT, P. (1990). Les nouvelles perspectives d'évaluation de l'intervention auprès des bénéficiaires, CQRS.

MERCIER, C. (1996). " La contribution de l'évaluation au renouvellement des pratiques d'intervention en santé mentale et en toxicomanie ", dans CQRS, Évaluer : pourquoi? Actes du colloque du Conseil québécois de la recherche sociale, Chicoutimi, mai 1995, p. 87-108.

MIDY, F., C. VANIER et M. GRANT (1998). Guide d'évaluation participative et de négociation, Montréal, UQAM.

MOORE, D., D. Gagnon et A. PeRREAULT (1998). Stratégie de travail de rue, Pertinence et recommandations, Régie régionale de la santé et des services sociaux, Montérégie, $32 \mathrm{p}$.

Ministère de LA SANTÉ ET DES SERVICES SOCIAUX (1995). Priorités nationales de santé publique : 1997-2002, Québec, 103 p.

MINISTĖRE DE LA SANTÉ ET DES SERVICES SOCIAUX (1992). La politique de la santé et du bien-être, Québec, $192 \mathrm{p}$.

NOËL, L., F. MiCHAUD et G. MARQUIS (1994). Rapport d'évaluation de Point de Repères, Période d'avril 1992 à mars 1993, Centre de santé publique de Québec.

PAQUET, M. (1998). Le travail de rue à Joliette : Évaluation du partenariat et étude exploratoire des effets sur la clientèle, Association pour les jeunes de la rue à Joliette et Direction de la santé publique, Régie régionale de la santé et des services sociaux de Lanaudière. 
PARAZELLI, M. (1990). « Pour ajouter de la misère à la vie : L'impact d'une épidémiologie sociale-étatique sur l'action communautaire et les problèmes sociaux ", Service social, vol. 39, no 2, p. 175-187.

PATton, M.P. (1986). Utilization-focused Evaluation, Beverly Hills, Sage, 368 p.

PHARAND, S. (1995). Le travail de rue au Québec, Revue documentaire, Rimouski, Unité de santé publique du Centre hospitalier régional de Rimouski, $52 \mathrm{p}$.

RÉGIE RÉGIONALE DE LA SANTÉ ET DES SERVICES SOCIAUX DE L'ABITIBITÉMISCAMINGUE (1999). Bilan de l'implantation du cadre de référence pour le répit, le dépannage, le gardiennage et les activités de jour, Programmes déficience intellectuelle et déficience physique, Direction des programmes et des services, $121 \mathrm{p}$.

RÉGIE RÉGIONALE DE LA SANTÉ ET DES SERVICES SOCIAUX DE L'ABITIBITÉMISCAMINGUE (1998). Hospitalisations prolongées en psychiatrie et ressources pour la clientèle nécessitant une aide de longue durée, Direction des programmes et des services, $86 \mathrm{p}$.

RÉGIE RÉGIONALE DE LA SANTÉ ET DES SERVICES SOCIAUX DE L'ABITIBITÉMISCAMINGUE (1996). Évaluation de la gestion des ressources de type familial, Direction des programmes et des services, $145 \mathrm{p}$.

RÉGIE RÉGIONALE DE LA SANTÉ ET DES SERVICES SOCIAUX DE L'ABITIBITÉMISCAMINGUE (1995). Évaluation de l'implantation du milieu de jour en santé mentale dans la MRC Vallée-de-l'Or, Direction des programmes et des services, $90 \mathrm{p}$.

SCHOOL OF Public HEALTH et INSTITUTE FOR HEALTH POLICY STUdies (1993). The Public Health Impact of Needle Exchange Programs in the United States and Abroad, Centers of Disease Control and Prevention.

TABLE DES PRIORITÉS DE SANTÉ ET DE BIEN-ÊTRE DE LA CONFÉRENCE DES RÉGIES RÉGIONALES (1994). Cadre pour la fonction évaluation dans les régies régionales, Rapport du groupe de travail sur la fonction évaluation. 
NDLR: Dans l'article qui suit, il faudrait lire, en haut de page, "des interventions sociales ". 